\title{
Phenotype-genotype interactions on renal function in type 2 diabetes: an analysis using structural equation modelling
}

\author{
X. Y. Song • S. Y. Lee $\cdot$ R. C. W. Ma $\cdot$ W. Y. So $\cdot$ \\ J. H. Cai • C. Tam • V. Lam • W. Ying • M. C. Y. Ng • \\ J. C. N. Chan
}

Received: 30 December 2008 / Accepted: 30 March 2009/Published online: 29 May 2009

(C) Springer-Verlag 2009

\begin{abstract}
Aims/hypothesis Cardiovascular and renal diseases share common risk factors. We used structural equation modelling (SEM) to evaluate the independent and combined effects of phenotypes and genotypes implicated in cardiovascular diseases on renal function in type 2 diabetes.
\end{abstract}

Electronic supplementary material The online version of this article (doi:10.1007/s00125-009-1400-1) contains supplementary material, which is available to authorised users.

X. Y. Song $\cdot$ S. Y. Lee $\cdot$ J. H. Cai

Department of Statistics, The Chinese University of Hong Kong, Shatin,

Hong Kong SAR, People's Republic of China

R. C. W. Ma $\cdot$ W. Y. So $\cdot$ C. Tam $\cdot$ V. Lam $\cdot$ W. Ying $\cdot$

M. C. Y. Ng J. C. N. Chan $(\bowtie)$

Department of Medicine and Therapeutics,

The Chinese University of Hong Kong, Prince of Wales Hospital,

32 Ngan Shing Street, Shatin,

Hong Kong SAR, People's Republic of China

e-mail: jchan@cuhk.edu.hk

\section{J. H. Cai}

Department of Statistics,

School of Mathematics and Computational Science,

Sun Yat-sen University,

Guangzhou, People's Republic of China

J. C. N. Chan

Hong Kong Institute of Diabetes and Obesity,

The Chinese University of Hong Kong, Prince of Wales Hospital,

Shatin,

Hong Kong SAR, People's Republic of China

J. C. N. Chan

Li Ka Shing Institute of Health Sciences,

The Chinese University of Hong Kong, Prince of Wales Hospital,

Shatin,

Hong Kong SAR, People's Republic of China
Methods 1,188 type 2 diabetic patients were stratified into high-risk and low-risk groups according to bimodal distributions of the logarithmically transformed $\left(\log _{e}\right)$ urinary albumin:creatinine ratio and plasma creatinine levels. Models for these groups, comprising continuous and non-ranking categorical data, were developed separately to evaluate the inter-relationships among measured variables and latent factors using non-linear SEMs, Bayesian estimation and model selection as assessed by a goodness-of-fit statistic.

Results Inter-correlated measured variables (obesity, glycaemia, lipid, blood pressure) and variants of the genes encoding endothelial nitric oxide synthase (NOS), $\beta$ adrenergic receptor (ADRB), components of the reninangiotensin system (RAS) and lipid metabolism were loaded onto their respective latent factors of phenotypes and genotypes. In addition to direct and indirect effects, latent factors of obesity, lipid and BP interacted with latent factors of ADRB and RAS genotypes to influence renal function. Together with variants of the genes encoding peroxisome proliferator-activated receptor $\gamma$, atrial natriuretic peptide, adducin, $G$ protein $\beta_{3}$ subunit, epithelial sodium channel $\alpha$ subunit and matrix metallopeptidase 3, these parameters explained $39-80 \%$ of the variance in renal function in the high-risk and low-risk models.

Conclusions/interpretation SEM is a useful tool for confirming and quantifying multiple interactions of biological pathways with genetic determinants. The combined and interactive effects of blood pressure, lipid and obesity on renal function may have therapeutic implications, especially in type 2 diabetic individuals with genetic risk factors.

Keywords Genotypes · Phenotypes - Renal function . Structural equation modelling · Type 2 diabetes 


$\begin{array}{ll}\text { Abbreviations } \\ \text { ACR } & \text { Albumin:creatinine ratio } \\ \text { ADRB } & \beta \text {-Adrenergic receptor } \\ \text { I/D } & \text { Insertion/deletion } \\ \text { PCr } & \text { Plasma creatinine } \\ \text { PP } & \text { Posterior predictive } \\ \text { RAS } & \text { Renin-angiotensin system } \\ \text { SEM } & \text { Structural equation modelling } \\ \text { SNP } & \text { Single nucleotide polymorphism }\end{array}$

\section{Introduction}

Type 2 diabetes is characterised by the clustering of risk factors, including hypertension, dyslipidaemia, obesity and inflammation, which interact in a complex manner to cause widespread vascular dysfunction, resulting in microvascular and macrovascular complications. Adding to this complexity are the genetic determinants underlying these phenotypic traits, as there are genotype-genotype, genotype-phenotype and phenotype-phenotype interactions [1].

Despite the growing body of knowledge on the epidemiology, genetics, pathogenesis and treatment of diabetes and associated complications, the challenge lies in integrating them within a conceptual framework for testing to improve our understanding of the causes and consequences of diseases. Structural equation modelling (SEM) is a useful tool for assessing inter-relationships among observed variables and latent factors. Basic SEM involves two components. The first component is a factor analysis model that groups highly correlated measured variables to form latent factors, e.g. systolic and diastolic BP to form a latent BP factor, or BMI and WHR to form a latent factor of obesity, taking random errors into account. The second component is a regression model, which regresses key outcome variables with other explanatory measured variables and/or latent factors. Thus, a latent factor of 'renal function' comprising measured variables of plasma creatinine $(\mathrm{PCr})$ and urinary albumin:creatinine ratio $(\mathrm{ACR})$ can be regressed on latent factors of BP and obesity to study the effects of 'BP' and 'obesity' on 'renal function'. In practice, the factor analysis model, defined a priori based on evidence and theory, is for confirmatory analysis, while the regression model can be used for exploratory analysis with different equations involving explanatory regressors (Fig. 1).

Structural equation modelling, commonly employed in sociology and management sciences, is now increasingly used in the field of biomedicine [2, 3]. However, conventional SEM only deals with continuous variables and cannot adequately handle missing and non-ranking categorical variables such as alleles (a, A) and genotypes (aa, AA, aA), where coding of 0,1 and 2 for aa, $\mathrm{AA}$ and

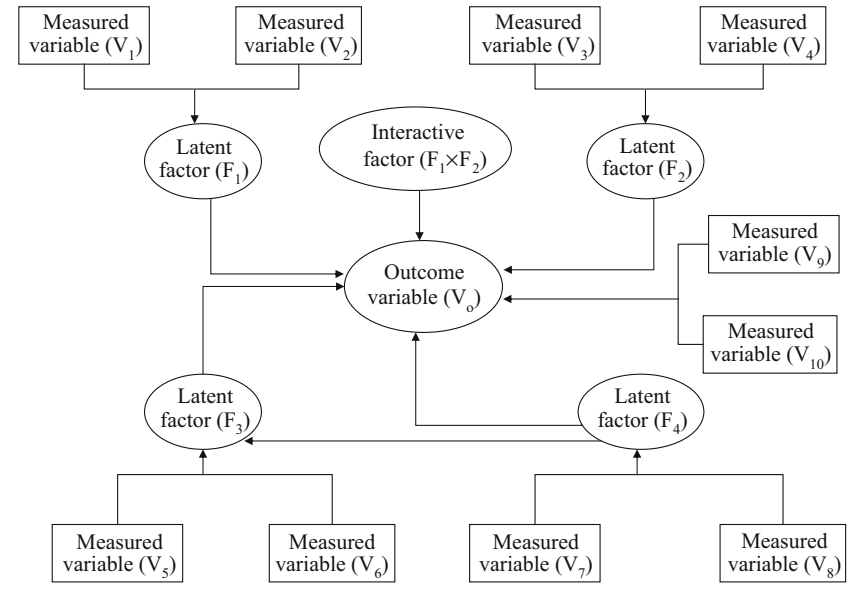

Fig. 1 The first component of SEM comprises a factor analysis model, in which closely correlated measured variables (in rectangles) are loaded onto latent factors (in ovals) to give a more precise interpretation. This is followed by regression of the outcome factors/ variables onto different factors/variables and their interaction terms. The strength of the relationships between the explanatory factors and outcome variable is indicated by the path coefficients

aA, respectively, does not imply $0<1<2$. Using a dataset consisting of genotypes and phenotypes, our group has refined the SEM methodology to include these types of data and has reported the interactive effects of genotypes and phenotypes on the risk of diabetic kidney disease [4].

Since cardiovascular and renal diseases share common risk factors, including obesity, hypertension and dyslipidaemia $[5,6]$, we examined the combined and interactive effects of genotypes and phenotypes of obesity, BP, lipid, endothelial function, stress/natriuretic responses, thrombosis and inflammation on renal function in type 2 diabetes. Using a panel of single nucleotide polymorphisms (SNPs) implicated in cardiovascular disease through dysregulation of lipid and homocysteine metabolism, endothelial function, thrombosis and coagulation, inflammation, stress responses and natriuresis (see Electronic supplementary material [ESM] Table 1) [7], we reported the risk associations of diabetic kidney disease with variants of genes encoding ACE, apolipoprotein E, apolipoprotein CIII, lipoprotein lipase and hepatic lipase in case-control and prospective cohorts [8-10].

In the present study, we tested the hypothesis that genetic variants may modify the expression of phenotypic traits of obesity, dyslipidaemia and hypertension to influence renal function (ESM Fig. 1) as follows: (1) dysregulation of the sympathetic nervous system causes obesity and dyslipidaemia [11]; (2) dysregulation of lipogenesis and lipoprotein metabolism causes ectopic fat deposition, dyslipidaemia, inflammation and insulin resistance [1215]; (3) abnormalities in stress and natriuretic responses and vascular reactivity contribute to hypertension [16, 17]; and 
Table 1 Distribution of phenotypes in 1,188 type 2 diabetic patients divided into two groups based on renal function

\begin{tabular}{|c|c|c|c|}
\hline Phenotypes & Whole group $(n=1,188)$ & High-risk group $(n=475)$ & Low-risk group $(n=713)$ \\
\hline Age (years) & $58(47-66)$ & $61(51-68)$ & $56(45-64)$ \\
\hline Duration of disease (years) & $8.0(2.0-13.0)$ & $8.0(2.8-12.0)$ & $8.0(2.0-13.0)$ \\
\hline Male & 42.0 & 45.6 & 39.7 \\
\hline Ex smoker & 13.4 & 17.7 & 10.6 \\
\hline Current smokers & 25.9 & 23.2 & 27.7 \\
\hline Systolic BP (mmHg) & $137(121-153)$ & $145(129-162)$ & $131(119-145)$ \\
\hline Diastolic BP (mmHg) & $79.1(71-86)$ & $81(74-90)$ & $78(70-85)$ \\
\hline Hypertension & 54.7 & 70.5 & 44.3 \\
\hline $\mathrm{HbA}_{1 \mathrm{c}}(\%)$ & $7.5(6.6-8.8)$ & $7.9(6.6-9.2)$ & $7.4(6.6-8.6)$ \\
\hline LDL-cholesterol (mmol/1) & $3.4(2.8-4.1)$ & $3.4(2.8-4.2)$ & $3.4(2.7-4.0)$ \\
\hline HDL-cholesterol (mmol/l) & $1.22(1.02-1.50)$ & $1.17(0.98-1.43)$ & $1.26(1.05-1.54)$ \\
\hline Triacylglycerol (mmol/l) & $1.31(0.90-1.96)$ & $1.51(1.01-2.14)$ & $1.18(0.82-1.81)$ \\
\hline Serum creatinine $(\mu \mathrm{mol} / \mathrm{l})$ & $75(61-95)$ & $87(68-128)$ & $69(58-84)$ \\
\hline $\operatorname{ACR}(\mathrm{mg} / \mathrm{mmol})$ & $2.56(0.89-41.87)$ & $49.5(8.9-186.2)$ & $1.19(0.72-3.01)$ \\
\hline Microalbuminuria $^{\mathrm{a}}$ & 18.9 & 23.4 & 15.9 \\
\hline Macroalbuminuria $^{\mathrm{b}}$ & 28.8 & 59.9 & 8.3 \\
\hline eGFR $\left(\mathrm{ml} \mathrm{min} \min ^{-1} 1.73 \mathrm{~m}^{-2}\right)$ & $110(84.8-110)$ & $90.8(56.5-120.2)$ & $117.4(99.6-138.1)$ \\
\hline eGFR $<60 \mathrm{ml} \mathrm{min}^{-1} 1.73 \mathrm{~m}^{-2}$ & 13.0 & 26.1 & 4.4 \\
\hline Retinopathy & 30.0 & 44.8 & 20.3 \\
\hline Sensory neuropathy & 27.3 & 38.6 & 19.8 \\
\hline Peripheral arterial disease ${ }^{c}$ & 9.5 & 13.8 & 6.6 \\
\hline History of stroke & 2.7 & 4.2 & 1.7 \\
\hline Lipid-lowering drugs & 7.1 & 11.0 & 4.5 \\
\hline Antihypertensive drugs & 28.4 & 41.2 & 20.0 \\
\hline $\mathrm{ACE}$ inhibitors or $\mathrm{ARB}$ & 11.2 & 16.3 & 7.7 \\
\hline Oral glucose-lowering drugs & 52.7 & 52.9 & 52.6 \\
\hline Insulin & 19.2 & 25.7 & 14.9 \\
\hline
\end{tabular}

Data are presented as median (interquartile range) or percentages

${ }^{a}$ Peripheral arterial disease was defined by an absent pedal pulse confirmed by an ankle:brachial ratio of $<0.9$

${ }^{\mathrm{b}}$ Microalbuminuria was defined as an ACR of $2.5-25 \mathrm{mg} / \mathrm{mol}$

${ }^{\mathrm{c}}$ Macroalbuminuria was defined as an ACR of $\geq 25 \mathrm{mg} / \mathrm{mol}$

$\mathrm{ARB}$, angiotensin II receptor blocker; eGFR, estimated GFR

(5) glucotoxicity, lipotoxicity, inflammation and haemodynamic abnormalities cause renal damage [18].

\section{Methods}

We examined these genotype-phenotype interactions on renal function in a consecutive cohort of 1,188 type 2 diabetic patients using the Hong Kong Diabetes Registry. None of the patients had a history of ketosis and none required insulin within the first year of diagnosis. All patients underwent a comprehensive assessment, including renal function measured by PCr and spot ACR. GFR was estimated using the modified Modification of Diet in Renal Disease (MDRD) equation for Chinese patients [19]. Diabetic kidney disease was defined as an estimated GFR of $<60 \mathrm{ml} \mathrm{min}^{-1} 1.72 \mathrm{~m}^{-2}$ [20]. All patients gave written informed consent and donated their DNA for research purposes. The study was approved by the Chinese University of Hong Kong Clinical Research Ethics Committee.

Genotyping DNA was extracted from whole blood using either a salting out [21] or a silica adsorption method (Nucleospin; Macherey-Nagel, Hoerdt, France). A total of 106 bi-allelic SNPs in 64 genes were genotyped in linear arrays provided by Roche Molecular Systems (Department of Human Genetics, Roche Molecular Systems, Alameda, CA, USA) [7]. These candidate genes were related to lipid and homocysteine metabolism, inflammation, thrombosis, endothelial function, stress and natriuretic responses (ESM 
Table 1) and were selected based on biological plausibility and risk associations with cardiovascular diseases using the Entrez Gene website (www.ncbi.nlm.nih.gov/sites/entrez? $d b=$ gene, accessed 1 June 2007).

Multiplex PCRs with biotinylated primers were used to amplify 106 SNPs in two reactions. The PCR products were hybridised against nylon membranes coated with immobilised oligonucleotides, which consisted of pairs of probes with sequences specific for the major or minor alleles. After conjugation with horseradish peroxidase and colour development with 3,3',5,5'-tetramethylbenzidine, the presence of a blue band indicated the presence of the respective allele. Genotypes were read by two independent investigators, and less than $2 \%$ of the genotypes could not be determined. Twenty-three SNPs with a heterozygote frequency of $<0.01$ were excluded, leaving 83 SNPs available for analysis.

Statistical methods The dataset contains continuous and ranking phenotype measurements, as well as non-ranking genotype measurements that do not allow the application of the classical SEM methods, such as those based on LISREL and/or PRELIS software [22]. In the present study, we applied the following statistical methods to increase the accuracy of the results and conclusions:

1. The histograms of $\log _{\mathrm{e}}(\mathrm{ACR})$ and $\log _{\mathrm{e}}(\mathrm{PCr})$ showed biomodal distributions. Patients were therefore separated into high-risk $(n=475)$ and low-risk $(n=713)$ groups using Bayesian classification based on a two-component mixture model [23] for independent analysis.

2. First, the data for the high-risk group was used to develop a model within the general framework of nonlinear SEMs comprising continuous and non-ranking categorical variables $[4,24]$, which is a generalisation of the well-known LISREL model [22]. In the factor analysis model, the inter-correlated measured variables were loaded onto latent factors, taking random errors into account. Then, in a posited regression model, we regressed the key outcome variable (latent factor of renal function) on explanatory variables, which can be direct or interaction terms comprising measured variables or latent factors (see Fig. 1). The magnitude of the path coefficients, analogous to the regression coefficients between independent and dependent variables in a regression model, indicated the strength of the relationships between the outcome and explanatory variables.

3. During modelling, the outcome variable (which can be a latent factor or measured variable, see Fig. 1) can be related with direct and interaction terms of the explanatory variables through different regression models. Of the regression models generated, the best is selected using the Bayes factor, a well-known Bayesian statistic for model selection $[4,25]$. Because of the complexity of the proposed non-linear SEM comprising interactions of latent factors and non-ranking categorical variables, classical tests such as the $\chi^{2}$ test and goodness-of-fit indices (e.g. Bentler-Bonett normed fit index) in existing software cannot be applied. We therefore used the posterior predictive (PP) $p$ value [26], which is a wellknown Bayesian model-checking statistic that measures the true discrepancy between the data and selected model to assess the goodness-of-fit of the selected model to the sample data. This statistic has been applied to many statistical models, including SEMs [24]. A PP $p$ value of between 0.4 and 0.6 indicates that the corresponding model fits the data well [26].

4. In the process leading up to the final model, we used the same factor analysis model, comprising the same measured phenotypes and genotypes, to identify latent factors. Different regression models were then considered by regressing the outcome variable on different direct and interaction terms of the explanatory variables comprising measured variables and latent factors. For example, we considered models that included the following interaction terms: latent factors of BP, glycaemia and obesity and genotypes of polymorphisms in the genes encoding endothelial nitric oxide synthase and proteins involved in the RAS and lipid metabolism. Each distinct regression model was considered a competing model to be selected by the Bayes factor. According to the common 'parsimonious' guideline, when comparing a model, $\mathrm{M}_{\mathrm{a}}$, with a simpler model, $\mathrm{M}_{\mathrm{b}}, \mathrm{M}_{\mathrm{b}}$ is selected if the corresponding Bayes factor statistic value does not give clear evidence to support $\mathrm{M}_{\mathrm{a}}$ according to the criterion provided by Kass and Raftery [25]. Since model selection is not the main theme of this paper, we have only presented the final models.

5. Statistical analyses similar to those described in step 2 were applied to other latent factors of phenotypes (dyslipidaemia, obesity and BP) to examine their relationships with renal function.

6. Steps $2-5$ were applied to the data for the low-risk group.

\section{Results}

Patients in the high-risk and low-risk groups had similar disease duration, but those in the former group were older and more likely to be male and ex-smokers (Table 1). They also had a higher BP, less favourable metabolic control and worse renal function and were more likely to have diabetic 
complications and receive insulin and/or cardio-protective drugs (Table 1).

Structural equation model on renal function in type 2 diabetes Figure 2 shows the path diagrams of the high-risk and low-risk models with the 'standardised' estimates of the path coefficients. The respective PP $p$ values of the models for the high-risk and low-risk groups were 0.549 and 0.488 , respectively, indicating a good fit of the data to the models. The models displayed in Fig. 2 represent the best models, which are substantially better than the models that poorly fitted the data, and better than models that barely fitted the data. Using data from the low-risk group (Fig. 2b) as an illustration, the Bayes factor statistic $\left(2 \log _{\mathrm{e}} \mathrm{B} 01\right)$ for comparing our selected model $\left(\mathrm{M}_{\mathrm{a}}\right)$ with a model $\left(\mathrm{M}_{\mathrm{b}}\right)$ that does not contain lipid or lipid $\times$ RAS as a latent factor is 20.93 , indicating that $M_{a}$ is substantially better, and the Bayes factor statistic for comparing $\mathrm{M}_{\mathrm{a}}$ with a model $\left(\mathrm{M}_{\mathrm{c}}\right)$ that does not contain glycaemia as a latent factor is 2.01 , indicating that $\mathrm{M}_{\mathrm{a}}$ is marginally better.

The latent factors in these selected models included phenotypes and genotypes (Table 2). The latent factors of phenotypes comprised renal function $(\log [\mathrm{PCr}]$ and $\log$ [urinary ACR]), obesity (WHR and BMI), BP (systolic and diastolic), lipids (fasting triacylglycerol and HDLcholesterol) and glycaemia $\left(\mathrm{HbA}_{1 \mathrm{c}}\right.$ and fasting plasma glucose). The latent factors of genotype included endothelial nitric oxide synthase (NOS), comprising the SNPs NOS3 $-922 \mathrm{~A}>\mathrm{G}$ and $1187 \mathrm{G}>\mathrm{T}$ (which were not in linkage disequilibrium), and RAS, comprising the insertion/deletion polymorphism within the angiotensin converting enzyme $(A C E \mathrm{I} / \mathrm{D})$ and a SNP in the gene encoding the angiotensin II receptor $(A G T R 11166 \mathrm{~A}>\mathrm{C})$. An interaction term of the latent factors of lipid phenotype and RAS genotype (lipid $\times$ RAS) also had a moderate effect on the outcome variable (latent factor of renal function) in both groups.

Because of marked differences in the scales of the observed variables, we standardised the variance of the latent factors and the results were presented based on the 'standardised solution' [22]. In the high-risk model, the latent factors collectively explained $37.40 \%$ of the variance of the dataset. The measured genotype of $M M P 3$, the independent latent factors of BP, obesity, lipid, glycaemia, NOS genotype and RAS genotype, together with the interaction term lipid $\times$ RAS, explained $38.80 \%$ of the variance of the outcome latent factor of renal function. The corresponding percentages for the low-risk model were $39.10 \%$ and $80.17 \%$.

We also treated each of the latent factors of obesity, lipid and BP as the outcome latent factor and examined the interrelationships between each phenotype factor and its related phenotypes or genotypes and their combined effects on renal function. Six submodels for three latent factors of phenotypes

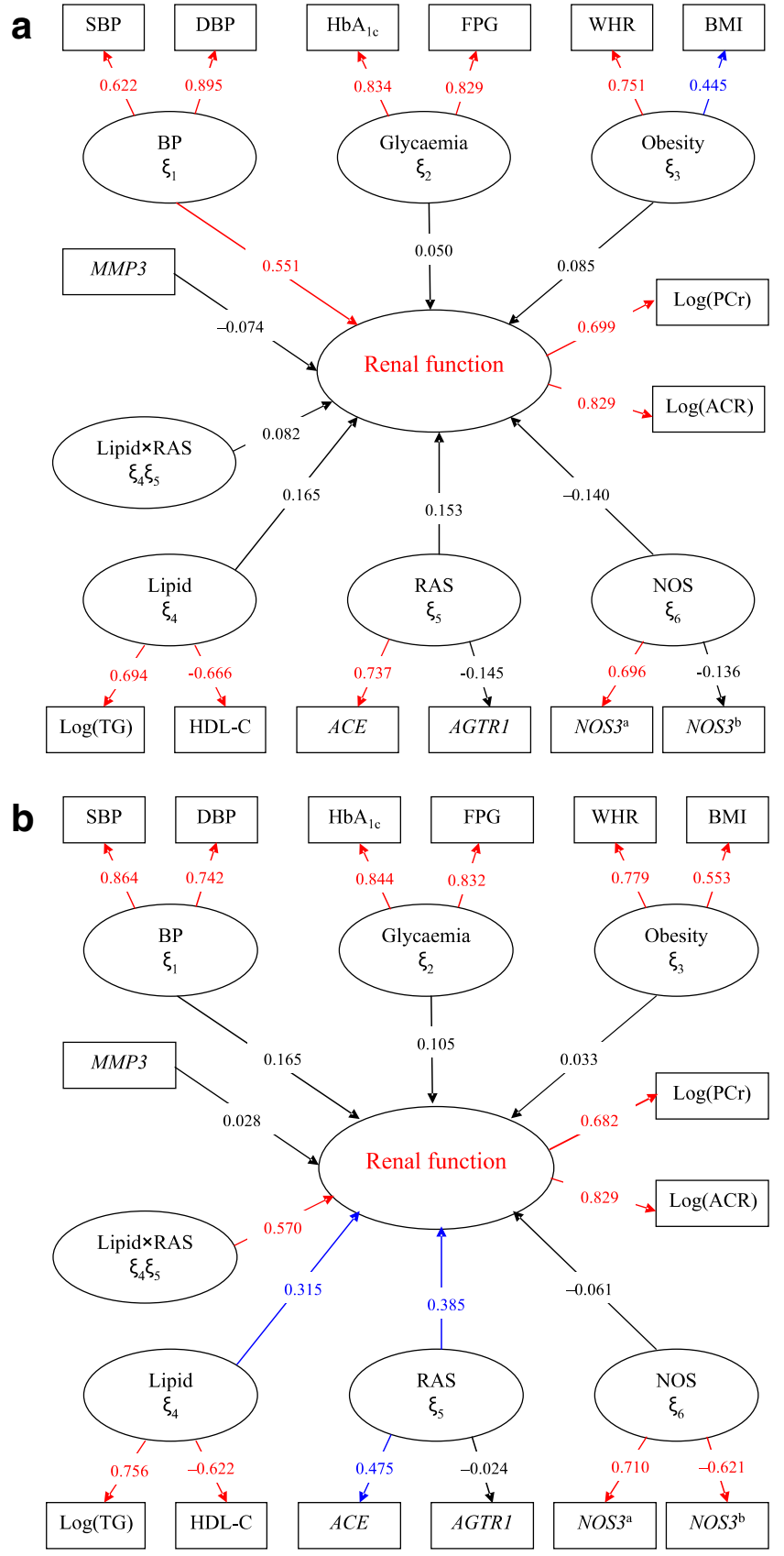

Fig. 2 A non-linear SEM showing interactions between measured variables (in rectangles) and latent factors (in ovals) on renal function in the high-risk (a) and low-risk (b) groups. The different coloured arrows indicate the size of the pathway coefficient: black line, $<0.2$; blue line, $0.2-0.5$; red line, $\geq 0.5$. The xi symbols $(\xi)$ indicate the individual latent factors and the interactive factor. Description of gene polymorphisms: $M M P 3, M M P 3$ promoter $5 \mathrm{~A} / 6 \mathrm{~A} ; A C E, A C E \mathrm{I} / \mathrm{D}$; AGTR1, AGTR1 1166A $>\mathrm{C} ; N O S 3^{\mathrm{a}}, N O S 3-922 \mathrm{~A}>\mathrm{G} ; N O S 3^{\mathrm{b}}, N O S 3$ $1187 \mathrm{G}>\mathrm{T}$. DBP, diastolic BP; FPG, fasting plasma glucose; HDL-C, HDL-cholesterol; NOS, endothelial nitric oxide synthase; SBP, systolic BP; TG, triacylglycerol

(obesity, BP and lipid) were developed for both the high-risk and low-risk groups (diagrams not shown). The PP $p$ values of these selected submodels ranged from 0.419 to 0.549 , supporting a good fit to the corresponding data. 
Table 2 Minor allele frequencies of SNPs with significant associations with renal function in type 2 diabetes, selected from a panel of 83 SNPs from 57 candidate genes encoding proteins involved in cardiovascular disease and inflammation

\begin{tabular}{|c|c|c|c|c|c|c|c|c|}
\hline \multirow{2}{*}{$\begin{array}{l}\text { Gene } \\
\text { symbol }\end{array}$} & \multirow[t]{2}{*}{ Gene name } & \multirow[t]{2}{*}{ Polymorphism } & \multirow[t]{2}{*}{ rs number } & \multirow[t]{2}{*}{ Chromosome } & \multirow[t]{2}{*}{ Position } & \multicolumn{3}{|c|}{ Minor allele frequency } \\
\hline & & & & & & $\begin{array}{l}\text { Whole } \\
\text { group }\end{array}$ & $\begin{array}{l}\text { High-risk } \\
\text { group }\end{array}$ & $\begin{array}{l}\text { Low-risk } \\
\text { group }\end{array}$ \\
\hline \multicolumn{9}{|c|}{ Lipid metabolism } \\
\hline$L I P C$ & Hepatic lipase & $-514 \mathrm{C}>\mathrm{T}$ & rs 1800588 & 15 & 56510967 & 0.378 & 0.397 & 0.366 \\
\hline$L P L$ & Lipoprotein lipase & $1719 \mathrm{C}>\mathrm{G}$ & rs 328 & 8 & 19864004 & 0.118 & 0.110 & 0.120 \\
\hline CETP & $\begin{array}{l}\text { Cholesteryl ester transfer } \\
\text { protein }\end{array}$ & $-628 \mathrm{C}>\mathrm{A}$ & rs 1800775 & 16 & 55552737 & 0.464 & 0.442 & 0.478 \\
\hline$P P A R G$ & $\begin{array}{l}\text { Peroxisome proliferator- } \\
\text { activated receptor } \gamma\end{array}$ & $125 \mathrm{C}>\mathrm{G}$ & rs1801282 & 3 & 12368125 & 0.028 & 0.029 & 0.027 \\
\hline \multicolumn{9}{|c|}{ Thrombosis/homocysteine metabolism } \\
\hline GNB3 & G protein $\beta_{3}$ subunit & $825 \mathrm{C}>\mathrm{T}$ & rs5443 & 12 & 6825136 & 0.446 & 0.462 & 0.436 \\
\hline \multicolumn{9}{|c|}{ Endothelial function/cytokine } \\
\hline \multirow[t]{2}{*}{ NOS3 } & \multirow{2}{*}{$\begin{array}{l}\text { Endothelial nitric oxide } \\
\text { synthase }\end{array}$} & $-922 \mathrm{~A}>\mathrm{G}$ & rs1800779 & 7 & 150127591 & 0.125 & 0.130 & 0.122 \\
\hline & & $1187 \mathrm{G}>\mathrm{T}$ & rs1799983 & 7 & 150133759 & 0.115 & 0.117 & 0.113 \\
\hline$L T A$ & Lymphotoxin $\alpha$ & $1069 \mathrm{~A}>\mathrm{G}$ & rs909253 & 6 & 31648292 & 0.440 & 0.452 & 0.432 \\
\hline \multicolumn{9}{|c|}{ Stress and natriuretic hormone } \\
\hline$A C E$ & $\begin{array}{l}\text { Angiotensin-converting } \\
\text { enzyme }\end{array}$ & Intron $16 \mathrm{I} / \mathrm{D}$ & rs 1799752 & 17 & 58919623 & 0.305 & 0.299 & 0.308 \\
\hline AGTRI & Angiotensin II receptor 1 & $1166 \mathrm{~A}>\mathrm{C}$ & rs5186 & 3 & 149942686 & 0.049 & 0.038 & 0.057 \\
\hline$A D R B 2$ & $\beta_{2}$-Adrenergic receptor & $285 \mathrm{~A}>\mathrm{G}$ & rs 1042713 & 5 & 148186633 & 0.399 & 0.384 & 0.409 \\
\hline$A D R B 3$ & $\beta_{3}$-Adrenergic receptor & $387 \mathrm{~T}>\mathrm{C}$ & rs 4994 & 8 & 37942955 & 0.124 & 0.126 & 0.122 \\
\hline$N P P A$ & Atrial natriuretic peptide & $664 \mathrm{G}>\mathrm{A}$ & rs 5063 & 1 & 11841914 & 0.133 & 0.128 & 0.136 \\
\hline$A D D 1$ & $\alpha$-Adducin & $1566 \mathrm{G}>\mathrm{T}$ & rs4961 & 4 & 2943715 & 0.404 & 0.415 & 0.397 \\
\hline SCNNIA & $\begin{array}{l}\text { Epithelial sodium channel } \alpha \\
\text { subunit }\end{array}$ & $2086 \mathrm{~A}>\mathrm{G}$ & rs2228756 & 12 & 6327325 & 0.448 & 0.450 & 0.446 \\
\hline$M M P 3$ & $\begin{array}{l}\text { Matrix metallopeptidase } 3 \\
\text { (stromelysin 1, progelatinase) }\end{array}$ & Promoter $5 \mathrm{~A} / 6 \mathrm{~A}$ & rs3025058 & 11 & 102221162 & 0.112 & 0.119 & 0.105 \\
\hline
\end{tabular}

Please refer to ESM Table 1 for the full list of SNPs

In the integrated path diagrams (ESM Fig. 2), which combined the results from the renal function model with those of the submodels of the three latent factors of phenotypes (obesity, BP and lipid), the determined genotypes of four polymorphisms, each in a separate gene ( $A D D 1$, $N P P A, G N B 3$ and $S C N N 1 A$ ), were found to have an effect on $B P$, which influenced renal function. Three lipid genotypes (LIPC, LPL and CETP) were loaded onto a latent factor of lipid genotypes, which influenced the latent factor of lipid phenotype. The genotype of a polymorphism in PPARG influenced both the lipid and obesity factors. The lipid latent factor also influenced the BP and obesity factors. The RAS genotype factor had direct effects on lipid, renal function and BP factors, and interacted with the lipid factor to influence renal function. Apart from the ADRB factor, an interaction term, lipid $\times$ ADRB, also had effect on the obesity factor. Table 3 summarises the path coefficients of the measured variables, associated latent factors and outcome variables in the integrated models of the high-risk and low-risk groups.
Comparisons between the high-risk and low-risk groups The high-risk and low-risk models shared similar measured variables, latent factors and interaction terms that explained the outcome variable of renal function in the main model, or obesity, BP or lipid in the submodels. Generally speaking, the explained variance was greater in the lowrisk than in the high-risk model. Most of the explanatory variables also had a greater effect on the outcome variable in the low-risk than in the high-risk model, but there were some exceptions.

Genotypes of the $M M P 3$ variant and the latent factors of NOS genotypes, obesity and BP all had greater effects, as indicated by the higher path coefficients, on renal function than they did in the low-risk model. In the submodels, the interaction term lipid $\times$ ADRB had a greater effect on the obesity factor in the high-risk than in the low-risk group. The lipid factor and several genotypes, including NPPA, $A D D 1$ and $G N B 3$, had greater effects on the BP factor in the high-risk than in the low-risk model (Table 3). 
Table 3 Summary of the path coefficients of the integrated models depicting the effects of phenotype, genotypes and their interactions on renal function in type 2 diabetic patients at high-risk or low-risk of renal dysfunction

\begin{tabular}{|c|c|c|c|c|c|}
\hline \multicolumn{3}{|c|}{ Factor analysis model } & \multicolumn{3}{|l|}{ Regression model } \\
\hline $\begin{array}{l}\text { Measured } \\
\text { variable }\end{array}$ & $\begin{array}{l}\text { Associate latent } \\
\text { factor }\end{array}$ & $\begin{array}{l}\text { Path } \\
\text { coefficient }\end{array}$ & $\begin{array}{l}\text { Explanatory latent or } \\
\text { observed variable }\end{array}$ & $\begin{array}{l}\text { Outcome latent } \\
\text { factor }\end{array}$ & $\begin{array}{l}\text { Path } \\
\text { coefficient }\end{array}$ \\
\hline \multicolumn{6}{|l|}{ High-risk group } \\
\hline $\log (\mathrm{PCr})$ & Renal function & 0.699 & $\mathrm{BP}$ & Renal function & 0.551 \\
\hline $\log (\mathrm{ACR})$ & & 0.829 & Glycaemia & & 0.050 \\
\hline Systolic BP & $\mathrm{BP}$ & 0.622 & Obesity & & 0.085 \\
\hline Diastolic BP & & 0.865 & Lipid & & 0.165 \\
\hline $\mathrm{HbA}_{1 \mathrm{c}}$ & Glycaemia & 0.834 & RAS & & 0.153 \\
\hline FPG & & 0.829 & NOS & & -0.140 \\
\hline WHR & Obesity & 0.751 & Lipid $\times$ RAS & & 0.082 \\
\hline BMI & & 0.445 & $M M P 3$ & & -0.074 \\
\hline $\log (\mathrm{TG})$ & Lipid & 0.694 & Lipid & $\mathrm{BP}$ & 0.178 \\
\hline HDL-C & & -0.666 & $N P P A$ & & -0.160 \\
\hline$A C E$ & RAS & 0.737 & $A D D 1$ & & 0.096 \\
\hline AGTR1 & & -0.145 & GNB3 & & 0.108 \\
\hline$N O S 3-922 \mathrm{~A}>\mathrm{G}$ & NOS & 0.696 & Lipid & Obesity & 0.452 \\
\hline NOS3 $1187 \mathrm{G}>\mathrm{T}$ & & -0.136 & ADRB & & -0.050 \\
\hline$A D R B 3$ & $\beta \mathrm{ADR}$ & 0.705 & Lipid $\times$ ADRB & & -0.339 \\
\hline$A D R B 2$ & & -0.203 & $P P A R G$ & & 0.053 \\
\hline$L I P C$ & Lipid genotype & 0.842 & Obesity & Lipid & 0.464 \\
\hline$L P L$ & & 0.133 & Lipid genotype & & -0.176 \\
\hline CETP & & 0.153 & $P P A R G$ & & -0.112 \\
\hline \multicolumn{6}{|l|}{ Low-risk group } \\
\hline $\log (\mathrm{PCr})$ & Renal function & 0.682 & $\mathrm{BP}$ & Renal function & 0.165 \\
\hline $\log (\mathrm{ACR})$ & & 0.829 & Glycaemia & & 0.105 \\
\hline Systolic BP & $\mathrm{BP}$ & 0.864 & Obesity & & 0.033 \\
\hline Diastolic BP & & 0.742 & Lipid & & 0.315 \\
\hline $\mathrm{HbA}_{1 \mathrm{c}}$ & Glycaemia & 0.844 & RAS & & 0.385 \\
\hline FPG & & 0.832 & NOS & & -0.061 \\
\hline WHR & Obesity & 0.779 & Lipid $\times$ RAS & & 0.570 \\
\hline BMI & & 0.553 & $M M P 3$ & & 0.028 \\
\hline $\log (\mathrm{TG})$ & Lipid & 0.756 & Lipid & $\mathrm{BP}$ & 0.060 \\
\hline HDL-C & & -0.622 & Obesity & & 0.356 \\
\hline$A C E$ & RAS & 0.475 & SCNN1A & & -0.059 \\
\hline AGTR1 & & -0.024 & Lipid & Obesity & 0.558 \\
\hline $\begin{array}{l}\text { NOS3 } \\
-922 \mathrm{~A}>\mathrm{G}\end{array}$ & NOS & 0.710 & ADRB & & -0.228 \\
\hline $\begin{array}{l}N O S 3 \\
1187 \mathrm{G}>\mathrm{T}\end{array}$ & & -0.621 & Lipid $\times$ ADRB & & -0.167 \\
\hline$A D R B 3$ & ADRB & 0.710 & PPARG & & -0.146 \\
\hline$A D R B 2$ & & 0.139 & Obesity & Lipid & 0.526 \\
\hline$L I P C$ & Lipid genotype & 0.802 & Lipid genotype & & -0.136 \\
\hline$L P L$ & & 0.142 & RAS & & -0.239 \\
\hline CETP & & -0.074 & PPARG & & -0.206 \\
\hline
\end{tabular}

See ESM Fig. 2 for the full models

FPG, fasting plasma glucose; HDL-C, HDL-cholesterol; TG, triacylglycerol 


\section{Discussion}

In this analysis, we examined the roles of genetic and modifiable risk factors in the public health problem of diabetic kidney disease using an advanced non-linear SEM [27]. We confirmed and quantified the complex genotypephenotype interactions and their combined effects on renal function in type 2 diabetes. The strengths of these relationships differed between the high-risk and low-risk models. In the low-risk group, the effects of lipid, RAS and lipid $\times$ RAS interaction and to a lesser extent, glycaemia explained most of the variance $(80 \%)$ of renal function. In the highrisk group, the BP phenotype, and to a lesser extent, obesity phenotype and NOS/MMP3 genotypes were important explanatory variables. In the submodels, the effects of interactions between lipid phenotype and ADRB genotypes on obesity phenotype were stronger in the high-risk than in the low-risk model. Furthermore, the lower percentage of explained variance for renal function $(39 \%)$ suggests that additional causal genotypes/phenotypes, especially those explaining the BP phenotype, will need to be discovered in the high-risk group. Taken together, our findings suggest that aggressive control of BP, obesity and associated dyslipidaemia, together with RAS inhibition, may have renoprotective effects, especially in individuals with genetic risks.

The novelty of our analysis lies in the demonstration of the totality of the effects of these genotypes and phenotypes simultaneously measured in a consecutive cohort of type 2 diabetic patients. Since many of these genetic variants have a population frequency of $10-50 \%$, and the explained variance of these combined models ranged from 39 to $80 \%$, the potential effects of the interactive effects of obesity, dyslipidaemia and BP on renal function can be substantial, especially in individuals carrying multiple genetic variants.

Application of SEM in diseases with multicausality Many common and complex diseases are caused by multiple interactions among genetic, environmental and/or acquired factors. Rothman et al. argued that, for a disease to become clinically manifest, there is a need for sufficient causes, made up of component causes acting in concert, although such a theory is difficult to prove using conventional experimental methods, each of which has its own limitations and biases [28]. SEM is an efficient statistical tool that enables us to examine various effects of explanatory factors on a key outcome variable in a model, supported by evidence and theory, inferred from other observations or experimental studies. It aims to complement other research methods to test a hypothesis in a holistic manner. Additionally, by including new paths or interaction terms during modelling, SEM is able to generate alternative hypotheses for further testing using appropriate experimental methods. Given the limitations of current research methods, SEM plays an important role by providing an integrated explanation of biological phenomena which cannot be easily tested in an experimental environment.

Effects of genotypes and phenotypes on renal function In this analysis, we have identified a bimodal distribution of two cohorts with distinct phenotypes characterised by different cardiovascular risk profiles, including renal dysfunction. In accordance with our current understanding [29, 30], we observed the important causal effects of lipid, RAS and glycaemia on renal function in the low-risk model, and of BP and NOS/MMP3 genotypes in the high-risk model. Several chromosomal regions linked to diabetic kidney disease contain candidate genes, including the CNDP1, ADIPOQ, ELMO1, SOD2, ACE and NOS3 [18]. In support of these findings, we have quantified the effects of the RAS and NOS genotypes on renal function in both models. We also confirmed the effects of genetic variants of $L P L$ and $L I P C$ on renal function [8-10], mediated via lipid phenotype, which in turn interacted with RAS genotype and BP and obesity phenotypes to influence renal function.

We also observed important differences in terms of the strength of these relationships, as indicated by the path coefficients and the percentage explained variance between the high-risk and low-risk models. The path coefficients of glycaemia (0.105 vs 0.05$)$ and RAS genotypes $(0.385$ vs 0.153$)$ were higher in the low-risk than in the high-risk model (Table 3). These findings are in line with the proven effectiveness of blood glucose control and RAS inhibition to halt the progression of albuminuria, or even induce remission, during early stages of the disease [31-33]. On the other hand, once clinical proteinuria and renal insufficiency sets in, control of BP ( 0.165 vs 0.551 in the low-risk and high-risk groups, respectively) assumes greater importance in determining the rate of deterioration in renal function [30]. Furthermore, obesity phenotype $(0.033$ vs 0.085$)$ (which was influenced by lipid $\times$ ADRB interaction $[-0.167$ vs $-0.339])$ and the NOS $(-0.061$ vs -0.14$)$ and $M M P 3$ $(-0.028$ vs -0.074$)$ genotypes appeared to be more important in the high-risk than in the low-risk group.

Interactive effects of RAS system and dyslipidaemia on renal function With the onset of proteinuria, there is further perturbation of lipid profiles, which can result in tubular interstitial inflammation and fibrosis [34, 35]. Although it is plausible that variants of genes encoding proteins involved in inflammatory pathways affect renal function, we were unable to confirm this, possibly as the result of an inadequate sample size, a small effect or measurement errors. However, we observed direct effects of lipids on renal function in both high-risk and low-risk models. Several proof-of-concept studies and post hoc analyses also sug- 
gested the potential benefits of lipid lowering, including the use of statins [36] and fibrates [37], on renal function.

More significantly, we identified an interaction effect between RAS genotype and lipid phenotype on renal function, and the respective path coefficients were 0.082 in the high-risk model and 0.570 in the low-risk model. In both human and animal studies, hypercholesterolaemia and triacylglycerolaemia can attenuate the vasodilator system (nitric oxide and eicosanoid production), activate the RAS, affect cellular membrane viscosity and modify membrane ion transport activity. These metabolic, cellular and vascular effects can lead to endothelial dysfunction with cardiorenal manifestations $[38,39]$. In light of this, there is some preliminary evidence supporting additive effects of RAS inhibition and lipid lowering on reducing the rate of decline of renal function [40].

Interactive effects of $\beta$-adrenergic activity and lipids on obesity In this integrated model, we confirmed the effects of variants of genes encoding proteins involved in multiple lipid pathways, notably lipolysis ( $L I P C$ and $L P L)$ and protein transfer $(C E T P)$, on lipid phenotypes typified by high triacylglycerol and low HDL-cholesterol levels [15]. The predictive effects of obesity on dyslipidaemia are in keeping with the known effects of increased portal transfer of NEFA to the liver in obesity, causing increased hepatic synthesis of lipoproteins. The consequent increase in triacylglycerol-rich lipoproteins and NEFA flux can accentuate hepatic glucose production, attenuate peripheral glucose uptake, worsen insulin resistance and alter the activities of hormonesensitive lipases, thus setting up a vicious cycle [41].

Complex interplay between BP, lipids and obesity on renal function Our integrated model also highlights close interactions amongst the lipid, BP and obesity phenotypes on renal function. These phenotypic traits, in turn, are influenced by the genotypes of variants in genes relevant to the lipid, RAS and $\beta$-adrenergic pathways. Thus, it can be inferred that these genetic variants and modifiable risk factors may interact in a multiplicative manner to establish a vicious cycle involving obesity, dyslipidaemia, hypertension and renal dysfunction. The interaction term of lipid $\times$ ADRB on obesity, especially in the highrisk model, is consistent with known risk associations of genetic variants of $A D R B 2$ and $A D R B 3$ with diabetes, obesity, fatty liver, dyslipidaemia, hypertension and reduced metabolic rate in multiple populations [42-46]. Since sympathetic adrenergic activity is a major determinant of lipolysis and energy metabolism, it is plausible that a combination of genetic factors that promote lipogenesis and/or reduce lipolysis may facilitate energy storage during times of deprivation but predispose to obesity during times of plenty [47].
Clinical meanings of the SEM These complex genotypephenotype interactions are compatible with our observations on the combined effects of central obesity, high triacylglycerol, low HDL-cholesterol and BP on the incidence of diabetic kidney disease in a 5 year prospective survey [48]. In another 18 year prospective analysis of a community-based cohort, age, BMI, diabetes, smoking and estimated GFR were major predictors of new-onset of chronic kidney disease [49]. Other prospective and clinical trials also confirmed the beneficial effects of BP and lipid control in reducing or stabilising the rate of decline of renal function $[30,36]$. Given the considerable residual risk of end-stage renal disease in type 2 diabetic individuals, despite receiving the most effective therapies, including RAS inhibition and control of BP, glycaemia and hypercholesterolaemia [50], our findings suggest that control of obesity and associated dyslipidaemia, together with aggressive blockade of RAS, may confer further renoprotection. These benefits may be particularly relevant in those with a genetic predisposition to renal dysfunction, especially during early stages of the disease, as indicated by the explained variance of nearly $80 \%$ in the low-risk model. On the other hand, the lower explained variance of $39 \%$ in the high-risk model suggests that additional factors, such as genotypes/phenotypes regulating BP, inflammation, cellular growth, energy metabolism and homeostasis may need to be identified.

Application and limitations of the current SEM In this novel analysis, we have applied an advanced non-linear SEM with non-ranking categorical variables to a convenient cohort with available genotypes and phenotypes, to form latent factors of genotypes and phenotypes in order to examine their direct and interactive effects on renal function in type 2 diabetes. Among a number of competing models comprising explanatory measured variables and latent factors and their interaction terms, we used Bayes factor to select the most appropriate model [25]. Like all statistical methods, SEM has its limitations, such as the assumption of normality of latent factors and error terms in the model. Our group has recently developed a semiparametric Bayesian method [51] that does not require the normality assumption. Moreover, our current SEM cannot be used to assess changes in longitudinal effects over time; this would require the extension of dynamic SEMs [4, 52] to include non-ranking categorical variables. Another limitation is the need for a sufficiently large sample size. We expect our methodologies to be sensitive to the low number of individuals in some cells in the contingency table associated with non-ranking categorical variables. In this situation, we ensured the convergence of the Markov chain Monte Carlo (MCMC) algorithm by running multiple iterations and checking whether the same results (in 
variable estimation and model selection) were obtained when using different starting values.

\section{Conclusion}

As our understanding of disease pathogenesis and mechanisms increases through the use of genetic, genomic, biological and clinical approaches, SEM which allows quantitative estimation of the interactions between latent factors of both phenotypes and genotypes will be a useful tool for researchers to analyse data in an integrated manner for clinical application.

Acknowledgements The research of X. Y. Song, S. Y. Lee, J. H. Cai, and J. C. N. Chan was partially supported by a Research Fund for the Control of Infectious Diseases (no. 07060312) awarded by the Health, Welfare and Food Bureau of the Hong Kong SAR and the Lioa Wun Yuk Diabetes Memorial Research Fund of the Chinese University of Hong Kong. We thank all the medical and nursing staff at the Prince of Wales Diabetes and Endocrine Centre for recruiting and managing the patients. Special thanks are extended to K. Lindpaintner and S. Cheng (Roche) for providing the line arrays for genotyping.

Duality of interest J. C. N. Chan has filed a patent application on behalf of the Chinese University of Hong Kong to use genetic variants to predict the risk of diabetic kidney disease in Chinese populations. None of the other authors has a duality of interest.

\section{References}

1. Van Gaal LF, Mertens IL, de Block CE (2006) Mechanisms linking obesity with cardiovascular disease. Nature 444:875880

2. Chan JCN, Cheung JCK, Lau EMC, Woo J, Swaminathan R, Cockram CS (1996) The metabolic syndrome in Hong Kong Chinese. The inter-relationships amongst its components analysed by structural equation modeling. Diabetes Care 19:953-959

3. Chan JCN, Cheung JCK, Stehouwer CDA et al (2002) The central roles of obesity-associated dyslipidaemia, endothelial activation and cytokines in the metabolic syndrome - an analysis by structural equation modelling. Int J Obes 26:994-1008

4. Song XY, Lee SY, Ng MC, So WY, Chan JC (2007) Bayesian analysis of structural equation models with multinomial variables and an application to type 2 diabetic nephropathy. Stat Med 26:23482369

5. Libby P, Theroux P (2005) Pathophysiology of coronary artery disease. Circulation 111:3481-3488

6. Schrijvers BF, de Vriese AS, Flyvbjerg A (2004) From hyperglycemia to diabetic kidney disease: the role of metabolic, hemodynamic, intracellular factors and growth factors/cytokines. Endocr Rev 25:971-1010

7. Ng MCY, Wang Y, So WY et al (2004) Ethnic differences in the linkage disequilibrium and distribution of single nucleotide polymorphisms in 35 candidate genes for cardiovascular diseases. Genomics 83:559-565

8. Ng MC, Baum L, So WY et al (2006) Association of lipoprotein lipase S447X, apolipoprotein E exon 4, and apoC3-455T $\rightarrow \mathrm{C}$ polymorphisms on the susceptibility to diabetic nephropathy. Clin Genet 70:20-28

9. Wang Y, Ng MCY, So WY et al (2004) Prognostic effect of insertion/deletion polymorphism of the ACE gene on renal and cardiovascular clinical outcomes in Chinese patients with type 2 diabetes. Diabetes Care 28:348-354

10. Baum L, Ng MC, So WY et al (2005) Effect of hepatic lipase $-514 \mathrm{C} \rightarrow \mathrm{T}$ polymorphism and its interactions with apolipoprotein $\mathrm{C} 3-482 \mathrm{C} \rightarrow \mathrm{T}$ and apolipoprotein $\mathrm{E}$ exon 4 polymorphisms on the risk of nephropathy in Chinese type 2 diabetic patients. Diabetes Care 28:1704-1709

11. Ishiyama-Shigemoto S, Yamada K, Yuan X, Ichikawa F, Nonaka K (1999) Association of polymorphisms in the $\beta 2$-adrenergic receptor gene with obesity, hypertriglyceridaemia, and diabetes mellitus. Diabetologia 42:98-101

12. Sharma AM, Staels B (2007) Review: peroxisome proliferatoractivated receptor $\gamma$ and adipose tissue-understanding obesityrelated changes in regulation of lipid and glucose metabolism. J Clin Endocrinol Metab 92:386-395

13. Permana PA, Menge C, Reaven PD (2006) Macrophage-secreted factors induce adipocyte inflammation and insulin resistance. Biochem Biophys Res Commun 341:507-514

14. Ahima RS, Flier JS (2000) Adipose tissue as an endocrine organ. Trends Endocrinol Metab 11:327-332

15. Lopez-Miranda J, Williams C, Lairon D (2007) Dietary, physiological, genetic and pathological influences on postprandial lipid metabolism. Br J Nutr 98:458-473

16. Luft FC (2004) Present status of genetic mechanisms in hypertension. Med Clin North Am 88:1-18 vii

17. Henrion D, Benessiano J, Iglarz M, Philip I, Levy BI (2002) Genetic determinants of vascular reactivity. Curr Hypertens Rep 4:41-48

18. Freedman BI, Bostrom M, Daeihagh P, Bowden DW (2007) Genetic factors in diabetic nephropathy. Clin J Am Soc Nephrol 2:1306-1316

19. Ma Y-C, Zuo L, Chen J-H et al (2006) Modified glomerular filtration rate estimating equation for Chinese patients with chronic kidney disease. J Am Soc Nephrol 17:2937-2944

20. Yang X, So WY, Tong PC et al (2008) Development and validation of an all-cause mortality risk score in type 2 diabetes. Arch Intern Med 168:451-457

21. Sambrook J, Fritsch E, Maniatis T (eds) (1989) Molecular cloning: a laboratory manual, 2nd edn. Cold Spring Harbor Laboratory Press, New York, pp E3-E4

22. Jöreskog KG, Sörbom D (eds) (1996) LISREL 8: structural equation modeling with the SIMPLIS command language. Scientific Software International, London

23. Titterington DM, Smith AFM, Markov UE (1985) Statistical analysis of finite mixture distributions. Wiley, Chichester

24. Lee SY (ed) (2007) Structural equation modelling: a Bayesian approach. Wiley, Chichester

25. Kass RE, Raftery AE (1995) Bayes factors. J Am Stat Assoc 90:773-795

26. Gelman A, Meng XL, Stern H (1996) Posterior predictive assessment of model fitness via realized discrepancies. Statistica Sinica 6:773-807

27. Yamagishi S, Fukami K, Ueda S, Okuda S (2007) Molecular mechanisms of diabetic nephropathy and its therapeutic intervention. Curr Drug Targets 8:952-959

28. Rothman KJ, Greenland S (2005) Causation and causal inference in epidemiology. Am J Public Health 95(Suppl 1):S144-S150

29. Keane WF, Brenner BM, de Zeeuw D et al (2003) The risk of developing end-stage renal disease in patients with type 2 diabetes and nephropathy: the RENAAL study. Kidney Int 63:1499-1507

30. Bakris GL, Williams M, Dworkin L et al (2000) Preserving renal function in adults with hypertension and diabetes: a consensus 
approach. National Kidney Foundation Hypertension and Diabetes Executive Committees Working Group. Am J Kidney Dis 36:646-661

31. Holman RR, Paul SK, Bethel MA, Matthews DR, Neil HA (2008) 10-year follow-up of intensive glucose control in type 2 diabetes. N Engl J Med 359:1577-1589

32. Patel A, MacMahon S, Chalmers J et al (2008) Intensive blood glucose control and vascular outcomes in patients with type 2 diabetes. N Engl J Med 358:2560-2572

33. Parving HH, Lehnert H, Brochner-Mortensen J et al (2001) The effect of irbesartan on the development of diabetic nephropathy in patients with type 2 diabetes. N Engl J Med 345:870-878

34. Moorhead JF, Chan MK, El-Nahas M, Varghese Z (1982) Lipid nephrotoxicity in chronic progressive glomerular and tubulointerstitial disease. Lancet ii:1309-1311

35. Wong CK, Ho AW, Tong PC et al (2007) Aberrant activation profile of cytokines and mitogen-activated protein kinases in type 2 diabetic patients with nephropathy. Clin Exp Immunol 149:123-131

36. Fried L, Orchard T, Kasiske B, for the lipids and renal disease progression meta-analysis study group (2001) Effect of lipid reduction on the progression of renal disease: a meta analysis. Kidney Int 59:260-269

37. Ansquer J, Foucher C, Rattier S, Taskinen M, Steiner G, DAIS Investigators (2005) Fenofibrate reduces progression to microalbuminuria over 3 years in a placebo-controlled study in type 2 diabetes: results from the Diabetes Atherosclerosis Intervention Study (DAIS). Am J Kidney Dis 45:485-493

38. Amaral AC, Sposito AC (2006) Lipid modulation of intravascular and cellular sodium handling: mechanistic insights and potential clinical implications. Curr Vasc Pharmacol 4:409-417

39. Chen J, Li D, Schaefer R, Mehta JL (2006) Cross-talk between dyslipidemia and renin-angiotensin system and the role of LOX-1 and MAPK in atherogenesis studies with the combined use of rosuvastatin and candesartan. Atherosclerosis 184:295-301

40. Cases A, Coll E (2005) Dyslipidemia and the progression of renal disease in chronic renal failure patients. Kidney Int Suppl: S87-S93
41. Adiels M, Olofsson SO, Taskinen MR, Boren J (2006) Diabetic dyslipidaemia. Curr Opin Lipidol 17:238-246

42. Iwamoto N, Ogawa Y, Kajihara S et al (2001) Gln27Glu beta2adrenergic receptor variant is associated with hypertriglyceridemia and the development of fatty liver. Clin Chim Acta 314:85-91

43. Fujisawa T, Ikegami H, Yamato E et al (1996) Association of Trp64Arg mutation of the beta3-adrenergic-receptor with NIDDM and body weight gain. Diabetologia 39:349-352

44. Sipilainen R, Uusitupa M, Heikkinen S, Rissanen A, Laakso M (1997) Polymorphism of the beta3-adrenergic receptor gene affects basal metabolic rate in obese Finns. Diabetes 46:77-80

45. Walston J, Silver K, Bogardus C et al (1995) Time of onset of non-insulin-dependent diabetes mellitus and genetic variation in the $\beta_{3}$-adrenergic-receptor gene. N Engl J Med 333:343-347

46. Thomas GN, Tomlinson B, Chan JCN, Young RP, Critchley JAJH (2000) The Trp64Arg polymorphism of the $\beta_{3}$-adrenergic receptor gene and obesity in Chinese subjects with components of the metabolic syndrome. Int J Obes 24:545-551

47. Neel JV (1962) Diabetes mellitus: a "thrifty" genotype rendered detrimental by "progress"? Am J Hum Genet 14:353-362

48. Luk AO, So WY, Ma RC et al (2008) Metabolic syndrome predicts new onset of chronic kidney disease in 5,829 patients with type 2 diabetes: a 5-year prospective analysis of the Hong Kong Diabetes Registry. Diabetes Care 31:2357-2361

49. Fox CS, Larson MG, Leip EP, Culleton B, Wilson PW, Levy D (2004) Predictors of new-onset kidney disease in a communitybased population. JAMA 291:844-850

50. Chan JCN, Wat NMS, So WY et al (2004) RAAS blockade and renal disease in type 2 diabetic patients: an Asian perspective from the RENAAL Study. Diabetes Care 27:874-879

51. Lee SY, Lu B, Song XY (2008) Semiparametric Bayesian analysis of structural equation models with fixed covariates. Stat Med 27:2341-2360

52. Song XY, Lee SY, Hser YI (2009) Bayesian analysis of multivariate latent curve models with nonlinear longitudinal latent effects. Structural Equation Modeling 16:245-266 